\title{
Quantifying Artifacts in Ewald Simulations of Inhomogeneous Systems with a Net Charge
}

\author{
Jochen S. Hub, ${ }^{* \dagger}$ Bert L. de Groot, ${ }^{\ddagger}$ Helmut Grubmüller, ${ }^{\ddagger}$ and Gerrit Groenhof ${ }^{*, \neq, \$}$ \\ ${ }^{\dagger}$ Institute for Microbiology and Genetics Georg-August-Universität Göttingen Justus-von-Liebig-Weg 11, 37077 Göttingen, Germany \\ *Computational biomolecular dynamics group, Max-Planck-Institute for Biophysical Chemistry Am Fassberg 11, 37077 Göttingen, \\ Germany \\ ${ }^{\S}$ Department of Chemistry and Nanoscience Center University of Jyväskylä P.O. Box 35, 40014 Jyväskylä, Finland
}

\begin{abstract}
Ewald summation, which has become the de facto standard for computing electrostatic interactions in biomolecular simulations, formally requires that the simulation box is neutral. For non-neutral systems, the Ewald algorithm implicitly introduces a uniform background charge distribution that effectively neutralizes the simulation box. Because a uniform distribution of counter charges typically deviates from the spatial distribution of counterions in real systems, artifacts may arise, in particular in systems with an inhomogeneous dielectric constant. Here, we derive an analytical expression for the effect of using an implicit background charge instead of explicit counterions, on the chemical potential of ions in heterogeneous systems, which (i) provides a quantitative criterium for deciding if the background charge offers an acceptable trade-off between artifacts arising from sampling problems and artifacts arising from the homogeneous background charge distribution, and (ii) can be used to correct this artifact in certain cases. Our model quantifies the artifact in terms of the difference in charge density between the non-neutral system with a uniform neutralizing background charge and the

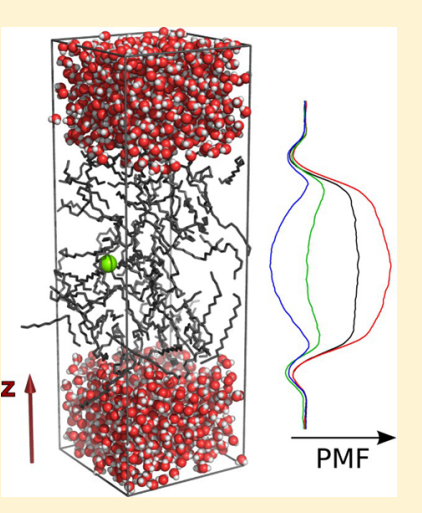
real neutral system with a physically correct distribution of explicit counterions. We show that for inhomogeneous systems, such as proteins and membranes in water, the artifact manifests itself by an overstabilization of ions inside the lower dielectric by tens to even hundreds kilojoules per mole. We have tested the accuracy of our model in molecular dynamics simulations and found that the error in the calculated free energy for moving a test charge from water into hexadecane, at different net charges of the system and different simulation box sizes, is correctly predicted by the model. The calculations further confirm that the incorrect distribution of counter charges in the simulation box is solely responsible for the errors in the PMFs.
\end{abstract}

\section{INTRODUCTION}

Molecular dynamics (MD) simulations have come of age. Since the first applications of MD on protein systems more than three decades ago, ${ }^{1,2}$ advances in computer power, algorithmic developments, and improvements in the accuracy of the applied interaction functions have established $\mathrm{MD}$ as an important predictive technique to study dynamic processes at atomic resolution. ${ }^{3,4}$ An important improvement in accuracy is achieved by the use of lattice summation methods for the evaluation of the Coulombic interactions in simulation boxes subject to periodic boundary conditions. In such approaches, the Coulomb interactions between all pairs of charged particles are accounted for, including interactions between the charges in the central box and their periodic images,

$$
V=\frac{1}{2} \sum_{i, j=1}^{N} \sum_{\mathbf{n} \in \mathbb{Z}^{3}} \frac{q_{i} q_{j}}{\mathrm{r}_{i j, \mathbf{n}}}
$$

where we used Gaussian units, as in the remainder of this article. Here, $N$ is the number of charged particles in the central simulation box, $\mathbf{n}=\left(n_{x}, n_{y}, n_{z}\right)$ is the box index vector. The prime indicates that if $i=j$ the term $\mathbf{n}=\mathbf{0}$ should be omitted. The distance $r_{i j, \mathbf{n}}$ is the real distance between the charges and not the distance to the nearest periodic image. Due to the longrange nature of the Coulomb interaction, this sum is only conditionally convergent, ${ }^{5}$ and it converges very slowly. By using the decomposition $1 / r=\operatorname{erfc}(\alpha r) / r+\operatorname{erf}(\alpha r) / r$, the Ewald summation speeds up the convergence by transforming the slowly converging series (1) into a short-range term that converges quickly in real space, a long-range smoothly varying term that converges quickly in reciprocal space, and a correction term: ${ }^{6-8}$

$$
\begin{aligned}
& V=V_{\text {dir }}+V_{\text {rec }}+V_{0} \\
& V_{\text {dir }}=\frac{1}{2} \sum_{i, j=1}^{N} \sum_{\mathbf{n} \in \mathbb{Z}^{3}}{ }^{\prime} q_{i} q_{j} \frac{\operatorname{erfc}\left(\alpha r_{i j, \mathbf{n}}\right)}{r_{i j, \mathbf{n}}} \\
& V_{\text {rec }}=\frac{1}{2 V_{\text {box }}} \sum_{\mathbf{k} \neq 0} \frac{4 \pi}{k^{2}} e^{-k^{2} / 4 \alpha^{2}}|\tilde{\rho}(\mathbf{k})|^{2}
\end{aligned}
$$

Received: July 18, 2013

Published: December 17, 2013 


$$
V_{0}=-\frac{\alpha}{\sqrt{\pi}} \sum_{i=1}^{N} q_{i}^{2}
$$

where $V_{\text {box }}$ is the volume of the unit cell, $\alpha$ the Ewald splitting parameter that determines the relative contributions of the direct and reciprocal sums, and $\mathbf{k}=\left(k_{x}, k_{y}, k_{z}\right)$ a vector in Fourier space. The Fourier transform of the real-space charge density $\rho(\mathbf{r})=\sum_{i=1}^{N} q_{i} \delta\left(\mathbf{r}-\mathbf{r}_{i}\right)$ is given by

$$
\tilde{\rho}(\mathbf{k})=\int_{V_{\mathrm{box}}} \mathrm{d} \mathbf{r} \rho(\mathbf{r}) \mathrm{e}^{-i \mathbf{k r}}=\sum_{j=1}^{N} q_{j} \mathrm{e}^{-i \mathbf{k} \mathbf{r}_{j}}
$$

Here, we only consider the case of so-called tinfoil boundary conditions, that is, our periodic lattice is surrounded by a conducting medium. Otherwise, also a correction term for the box dipole would be required. ${ }^{9}$ Although originally introduced as a means to compute the energy of a infinite ionic crystal, ${ }^{6}$ the Ewald technique is commonly used for simulations of noncrystalline systems as well.

If the real and reciprocal sums are sufficiently converged, the electrostatic energy is independent of $\alpha$ for neutral simulation boxes. If the simulation box is not neutral, an additional correction term is required to guarantee that the electrostatic energy does not depend on the choice of $\alpha$, which is discussed below. In practice, both sums are truncated such that the overall accuracy is below a given threshold. If, on the one hand, $\alpha$ is chosen such that the real space contribution vanishes at half the simulation box length, the direct sum includes all minimum image pairs and scales as $N^{2}$. In this situation, the number of terms needed in the reciprocal sum is of order $N$. If, on the other hand, $\alpha$ is chosen such that the real-space contribution vanishes at a predefined cutoff, which is typically much shorter than half the box length, the direct sum is of order $N$, but now the reciprocal sum is of order $N^{2}$. The optimal scaling, which can be achieved by varying $\alpha$, is of order $N^{3 / 2},{ }^{10}$ which precludes the application of the standard Ewald approach in simulations of large systems. As a consequence, widespread use of the Ewald summation technique had to await the development of more efficient grid-based approaches with $N \ln N$ scaling, such as the particle-particle-particle-mesh (PPPM) ${ }^{11}$ particle-mesh Ewald (PME) ${ }^{12}$ smooth particlemesh Ewald (SPME), ${ }^{13}$ or Fast Fourier Poission methods. ${ }^{14}$ Although originally developed for point charges, these methods have since then been extended to treat also point dipoles, ${ }^{15}$ higher order multipoles, ${ }^{16}$ continuous charge distributions and quantum mechanics/molecular mechanics (QM/MM) potentials. ${ }^{17,18}$ In addition, alternative approaches for treating longrange electrostatic interactions in molecular simulations have been developed, such as tree code, ${ }^{19}$ fast multipole, ${ }^{20}$ or isotropic sum methods. ${ }^{21}$ As the Ewald summation has remained the most popular method in biomolecular simulations, we have only analyzed artifacts associated with this method. All simulations in this work were conducted with the SPME method for computational efficiency, but the conclusions are valid for all variants of the Ewald technique.

Although the Ewald approach formally requires the system to be charge neutral, it can be applied to a charged system as well. As directly follows from eq 6 , the $k=0$ component of the density is equal to the sum of the charges in the simulation box:

$$
\tilde{\rho}(\mathbf{k}=0)=\sum_{i=0}^{N} q_{i}
$$

Leaving this term out upon back transformation causes a uniform shift in the (real-space) charge density:

$$
\rho^{\prime}(\mathbf{r})=\rho(\mathbf{r})-\sum_{i=0}^{N} q_{i} / V_{\text {box }}
$$

Thus, if the total charge is nonzero, the omission of the $k=0$ term introduces a uniform background (BG) charge density $\rho_{\mathrm{BG}}=-\sum_{i} q_{i} / V_{\mathrm{box}}$ in the box that effectively neutralizes the system. In that case, also a correction is required to account for interactions between the point charges and the background charge in the real space sum. ${ }^{22}$ The latter is achieved by shifting the electrostatic energy (eq 2) by a constant that depends on the Ewald splitting parameter:

$$
V_{\mathrm{n}}^{\mathrm{cor}}=-\frac{\pi\left(\sum_{i} q_{i}\right)^{2}}{2 \alpha^{2} V_{\mathrm{box}}}
$$

such that when averaged over the box volume, the Ewald potential is zero. This correction also ensures that the potential energy of the charged system becomes independent of a specific choice for $\alpha$.

Because the background charge is uniformly distributed and, hence, does not exert a force on charged particles, it is sometimes considered as a acceptable counter charge distribution. ${ }^{23}$ The background charge has an effect on the energy and pressure, but these artifacts can be corrected in homogeneous systems, either on-the-fly or a posteriori. ${ }^{24,25}$ Furthermore, it was shown that accurate hydration free energies of ions require only corrections for the finite volume of the ion and for interactions with the ion's periodic images and the background charge distribution. ${ }^{22,26-33}$ It may thus seem that, for a homogeneous system, the uniform background charge reflects a fully converged distribution of counterions and therefore offers a realistic and desirable setup. Indeed, several simulation studies have been reported in which the system was not neutralized by adding a suitable number of ions with opposite charge, but with the uniform background charge instead. In some cases, the use of the background charge distribution to neutralize the system may even be essential, as, for instance, (i) when calculating absolute solvation free energies of charged species by means of free energy perturbation techniques, (ii) if the ion parameters are not compatible with the protein or lipid force field ${ }^{34}$ or (iii) if the ion distribution converges too slowly.

The practice of neutralizing charged systems by the implicit background charge has not been limited to homogeneous systems with a single dielectric, but also heterogeneous systems with a nonhomogeneous dielectric constant, such as lipid bilayers in water, have been simulated this way. ${ }^{34}$ However, for systems with a nonhomogeneous dielectric constant, a uniformly distributed counter charge density is unphysical, because in reality the counterions would be found mostly inside the higher dielectric. Therefore, in contrast to homogeneous systems, artifacts may arise if a uniform background charge is used to neutralize nonhomogeneous systems. To decide whether also for nonhomogeneous systems the background charge offers a reasonable trade-off between sampling problems and accuracy, we have investigated the distribution of a charged particle in non-neutral inhomogeneous systems and compared the distributions to the situation in which explicit ions were added to neutralize these systems. As the partitioning of charges affects many important properties, such as protein 
stability, binding free energies or $\mathrm{p} K_{\mathrm{a}}$ values, we consider the partitioning of ions a crucial test for the validity of using a background charge to neutralize a system in MD simulations.

In the first part of this work, we introduce a theoretical model that quantifies the effect of a uniform background charge on the distribution of a test charge in a non-neutral heterogeneous system. The model, which is based on an analytical solution of the Poisson equation, demonstrates that the background charge may artificially cause the charged particle to favor the lower dielectric. The model can also quantitatively predict the effect of this artifact in realistic simulation systems, such as solvated lipid bilayers and proteins. In the second part, we tested the model with atomistic MD simulations. In these simulations, we have computed potentials of mean force (or free-energy profiles) associated with moving a charged particle from water with a high dielectric into a hexadecane slab with a low dielectric, using different numbers of positive and negative ions in the simulation boxes to create a net overall charge. The results of these simulations show that the uniform background charge distribution artificially changes the potential of mean force and that our theoretical model correctly quantifies this artifact. We have performed thermodynamic integration as well in order to validate this observation. Our simulations furthermore confirm that the artifact is not due to a direct interaction of the test particle with the background charge but due to an incorrect distribution of the charge in the box. Because the error in the partitioning of charged particles can be up to tens or even hundreds of kilojoules per mole, we recommend to neutralize the simulation box with explicit ions. We realize that the latter may not always be possible, for instance, if the total charge of the system changes during the simulation or if sampling the ions is computationally prohibitive. In these cases, however, our model not only provides a quantitative criterium to decide whether using the background is reasonable but also offers a means to correct for the artifacts a posteriori.

\section{METHODS}

Potentials of Mean Force (PMFs) for moving a test particle from water into a hexadecane slab were computed using the technique of umbrella sampling. ${ }^{35}$ For that purpose, a hexadecane/water simulation system box was taken from previous research, ${ }^{36}$ which contained $80 n$-hexadecane and 1104 TIP4P water molecules ${ }^{37}$ (Figure 1A). The system was equilibrated for $5 \mathrm{~ns}$ and simulated for another $15 \mathrm{~ns}$ under equilibrium conditions. In order to investigate the effect of the box size of a non-neutral system, the hexadecane/water box was extended in $z$-direction (perpendicular to the hexadecane layer) and filled with additional water, yielding systems containing 2348 and 4175 water molecules (Figures 1B/C). These systems were equilibrated for another 500 ps. The hexadecane molecules were modeled with the aliphatic atom types of the Berger et al. lipid force field. ${ }^{38}$ For the test particle, we used a potassium ion with $e / 4$ charge $(\sigma=0.49463 \mathrm{~nm}, \varepsilon=1.37234 \times$ $10^{-3} \mathrm{~kJ} / \mathrm{mol}$ ). The fractional charge was chosen to avoid that the ion drags water molecules into the hexadecane slab, as frequently occurs for ions with integer charge. Such dragging of water molecules may lead to very slowly converging PMFs.

We used two approaches to control the uniform background charge density. In the first approach, we used the smallest simulation system $1 \mathrm{~A}$ and varied the number of ions. In total, four PMFs were computed:

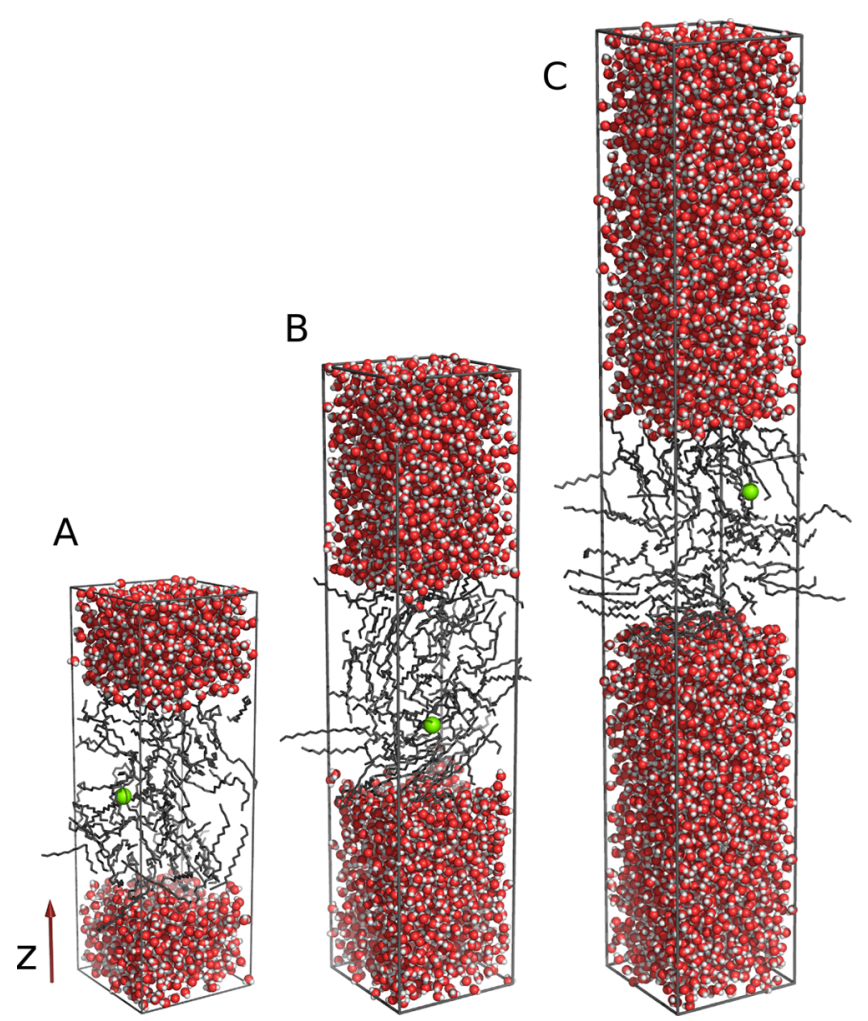

Figure 1. (A) Hexadecane/water simulation system. The probe particle of charge $+e / 4$ is shown as a green sphere. (B/C) Larger hexadecane/water simulation systems.

(i). with one $\mathrm{Na}^{+} \mathrm{Cl}^{-}$and neutralized with a counterion of $-e / 4$ (denoted hex-neutral, total system charge $Q=0$ );

(ii). with one $\mathrm{Cl}^{-}$ion (hex-1Cl, $\mathrm{Q}=-0.75 e$ );

(iii). with one $\mathrm{Na}^{+}$ion (hex-1Na, $Q=1.25 e$ ); and

(iv). with two $\mathrm{Na}^{+}$ions (hex-2Na, $Q=2.25 e$ ).

In addition, we also checked the influence of the spatial distribution of the counter charge distribution by

(a) mimicking the uniform background charge by adding small partial charges on all hexadecane and water atoms in the system, compensating the total net charge and thus creating a neutral system. For this purpose, the partial charges of all hexadecane and water atoms were changed by $\delta q_{\text {hex }}=-Q /\left(V_{\text {box }} \rho_{\text {hex }}\right)$ and $\delta q_{\mathrm{w}}=-Q /\left(V_{\text {box }} \rho_{\mathrm{w}}\right)$, respectively, where $\rho_{\text {hex }}$ and $\rho_{\mathrm{w}}$ denote the atom number density in the hexadecane and water phase, respectively. Note that this configuration represents an unphysical situation, because counter charge would under realistic conditions not be expected inside the hexadecane. We refer to this configuration as an explicit uniform background charge.

(b) creating an uniformly distributed counter charge only in the water phase by adding the partial charge $\delta q_{\mathrm{w}}=-\mathrm{Q}$ $N_{\mathrm{w}}$ to all water atoms, where $N_{\mathrm{w}}$ is the number of water atoms. We refer to this configuration as an explicit wateronly background charge.

By modifying the partial charges we change the force field model for the molecules. Although this might affect several properties, including the dielectric constant, we expect the effects, if any, to be very small, because the maximum changes in the partial charges amounts to $2 \times 10^{-4} e$. Furthermore, the perfect agreement between the simulation with the explicit 
background charge distribution, either on all atoms or on the water atoms only, and the corresponding simulations with an implicit background charge distribution or counterions, suggests that the effects are indeed negligible.

In the second approach, we used the box volume to control the density of the background charge. In these simulations, no additional ions were included, so that the background charge arises solely from the test particle itself. We varied the box volume by changing the box height $\left(L_{z}\right)$ from 8.8 to $13.2 \mathrm{~nm}$ and $19.78 \mathrm{~nm}$ (Figures $1 \mathrm{~A}-\mathrm{C}$ ). For comparison, we also computed the PMFs after neutralizing the boxes by adding the counterion with a charge of $-e / 4$.

All simulations were carried out using the Gromacs simulation software. ${ }^{39}$ Electrostatic interactions were calculated at every step with the SPME method, using a real-space cutoff at $1 \mathrm{~nm}$, a maximum spacing of $0.12 \mathrm{~nm}$ for the fast Fourier transform grid, a fourth-order (cubic) B-spline interpolation scheme, and tinfoil boundary conditions. ${ }^{12,13}$ Short-range repulsive and attractive dispersion interactions were described together by a Lennard-Jones potential, which was cut off at 1.0 $\mathrm{nm}$. The SETTLE ${ }^{40}$ algorithm was used to constrain bond lengths and angles of water molecules, and LINCS ${ }^{41}$ was used to constrain all other bond lengths, allowing a time step of $2 \mathrm{fs}$. The temperature was kept constant by coupling the system to a Nosé-Hoover thermostat $(\tau=2.5 \mathrm{ps}) .{ }^{42,43}$ In the simulations that were done for comparing the PMFs with the results from thermodynamic integration (TI), the volume was kept constant to facilitate convergence. All other PMFs were derived using the Parrinello-Rahman pressure coupling scheme which was only applied in the $z$-direction $(\tau=5 \mathrm{ps}) .{ }^{44}$ In simulations containing a counterion of charge $-e / 4$ to neutralize the test particle, the charge of one randomly selected water molecule was set to $-e / 4$ by adding $-e / 4$ to the oxygen atom. No atoms were restrained during the simulations (except for the test particle during umbrella samling). Simulation snapshots were taken at random times from equilibrium simulations and were subsequently used as starting configurations for the umbrella sampling simulations.

The hexadecane/water system was divided into $\sim 340$ sections with a distance of $0.25 \AA$ between adjacent sections. Each section corresponded to one umbrella window. For each umbrella window, the test particle was inserted into the structure close to the position corresponding to the minimum of the umbrella potential. During insertion, a distance of at least $1 \AA$ was kept to nearby atoms to ensure a successful energy minimization. The $z$-coordinate (membrane normal) of the particle was used as reaction coordinate, where $z=0$ corresponds to the center of mass of all hexadecane atoms. The particle was restrained with an harmonic umbrella potential $\left(k=800 \mathrm{~kJ} \mathrm{~mol}^{-1} \mathrm{~nm}^{-2}\right)$. Umbrella simulations for different box heights were carried out for 5000 ps, and all other umbrella simulations for 500 ps. The first 100 ps of each simulation was used for equilibration and the rest for analysis. The 340 umbrella histograms were collected from the simulations and the PMF was computed using a cyclic implementation of the weighted histogram analysis method (WHAM), ${ }^{45}$ as implemented in the g_wham software. ${ }^{46}$ The statistical uncertainty of the PMFs were estimated using the Bayesian bootstrap of complete histograms. ${ }^{46}$ That procedure does not require the calculation of accurate autocorrelation times but considers only complete histograms as independent data points, yielding a robust error estimate. The $67 \%$ confidence levels were typically $\sim 1.5$ and $0.4 \mathrm{~kJ} / \mathrm{mol}$ based on the 500-ps and 5000-ps simulations, respectively.

TI was used to compute the free energy for creating a test ion of charge $e / 4$ (a) at the center of the hexadecane slab or (b) at the center of the water slab. Accordingly, the position of the test ion was restrained in $z$ direction during TI simulations ( $k=$ $500 \mathrm{~kJ} \mathrm{~mol}^{-1} \mathrm{~nm}^{-2}$ ). Two sets of TI simulations were done. In the first set, no counterion was generated, and the system was hence neutralized by an implicit background charge. In the second set, the system was kept neutral by generating a counter charge of $-e / 4$ on the oxygen atom of a randomly chosen water molecule. Apart from the test ion and, optionally, the counterion, no other additional ions were present.

TI calculations were conduced in two steps: (i) turn on Lennard-Jones interactions between the test ion and all other atoms; (ii) turn on the Coulomb interactions. TI was carried out along an alchemical reaction coordinate $\lambda$, where $\lambda=0$ and $\lambda=1$ correspond to the initial (A) and final states (B), respectively. The potential energy function was linearly interpolated between these states: $V(\lambda)=(1-\lambda) V_{\mathrm{A}}+\lambda V_{\mathrm{B}}$. Step $i$ and ii were decomposed into 64 equally spaced $\lambda$-steps, and each $\lambda$-step was simulated for 2000 ps. The volume was kept constant, while all other simulation parameters were chosen as explained above. Free-energy differences were subsequently computed by integrating $\langle\partial V / \partial \lambda\rangle_{\lambda}$ from $\lambda=0$ to $\lambda=1$. Here, $\langle\cdot\rangle_{\lambda}$ denotes the average computed from the respective trajectory run at $\lambda$, after removing the first 200 ps for equilibration. Statistical errors for $\langle\partial V / \partial \lambda\rangle_{\lambda}$ were computed using binning analysis, ${ }^{47}$ which subsequently yields the error for $\Delta G$ via Gaussian error propagation.

\section{RESULTS}

Analytical Model for the Effect of the Charge Distribution on the PMF of a Test Charge. First, we derive an expression to quantify the effect of omitting explicit counterions on the potential of mean force (PMF) of a single test charge in a model system composed of a high and a low dielectric. Typical examples in a biological context would be a protein or a lipid bilayer solvated in water. We considered two situations. In the first situation, the test charge was neutralized by adding explicit ions inside the higher dielectric. In the second situation, a uniform background charge was used for neutralizing the system. We varied the position $\mathbf{x}_{0}$ of the test charge and computed the total electrostatic energy of these systems. Our calculations show that the Poisson equation, or, equivalently, Gauss' law, can be used to quantify the background charge effect in terms of the difference in charge density between these two systems. Because Gauss' law is sufficiently simple, it allows one to gain intuitive insight and to derive analytic expressions for the background charge effect for simple geometries.

For the charge densities of these two systems we take

$$
\begin{aligned}
& \rho_{1}\left(\mathbf{x} ; \mathbf{x}_{0}\right)=\rho_{c, 1}(\mathbf{x})+\rho_{t}\left(\mathbf{x} ; \mathbf{x}_{0}\right) \\
& \rho_{2}\left(\mathbf{x} ; \mathbf{x}_{0}\right)=\rho_{c, 2}(\mathbf{x})+\rho_{t}\left(\mathbf{x} ; \mathbf{x}_{0}\right)
\end{aligned}
$$

Here, the test charge density $\rho_{t}\left(\mathbf{x} ; \mathbf{x}_{0}\right)$ is localized around $\mathbf{x}_{0}$, and for a point-like test charge $q$ considered here, it is given by $\rho_{t}\left(\mathbf{x} ; \mathbf{x}_{0}\right)=q \delta^{3}\left(\mathbf{x}-\mathbf{x}_{0}\right)$. The densities $\rho_{c, 1}(\mathbf{x})$ and $\rho_{c, 2}(\mathbf{x})$ denote the counter charge distributions (including the background charge) that neutralize the systems, so that the total charge of the box is zero: $\int V_{\text {box }} \mathrm{d}^{3} \mathbf{x} \rho_{1,2}\left(\mathbf{x} ; \mathbf{x}_{0}\right)=0$. Although $\rho_{1,2}\left(\mathbf{x} ; \mathbf{x}_{0}\right)$ are 
kept general in the following calculation, $\rho_{c, 1}(\mathbf{x})$ and $\rho_{c, 2}(\mathbf{x})$ are typically the counter charge density of the implicitly and explicitly neutralized systems, respectively. Our key approximation is that the counter charge densities $\rho_{c, 1}$ and $\rho_{c, 2}$ do not depend on the position $\left(\mathbf{x}_{0}\right)$ of the test particle. We believe this assumption is valid, because the mobile charges that adapt to the position of the probe charge are inside the high-dielectric, where such charges do not build up a strong ionic atmosphere, due to shielding. We also do not consider the presence of an ionic atmosphere inside the lower dielectric, because of the high free energy penalty for transferring multiple ions from the higher into the lower dielectric medium. These assumptions are validated below by comparing the result to the computed potentials of mean force (PMFs).

Next, the difference between the two systems in terms of charge density, electrostatic potential, and potential energy of the test charge is considered. We introduce the charge density difference,

$$
\delta \rho(\mathbf{x}):=\rho_{1}\left(\mathbf{x} ; \mathbf{x}_{0}\right)-\rho_{2}\left(\mathbf{x} ; \mathbf{x}_{0}\right)=\rho_{c, 1}(\mathbf{x})-\rho_{c, 2}(\mathbf{x})
$$

which is independent of the test charge position $\mathbf{x}_{0}$. Furthermore, by concentrating on differences between the two systems, self-interaction effects due to periodicity will cancel, which facilitates the comparison. The difference between the electrostatic potentials of the two systems is

$$
\delta \Phi(\mathbf{x})=\Phi_{1}\left(\mathbf{x} ; \mathbf{x}_{0}\right)-\Phi_{2}\left(\mathbf{x} ; \mathbf{x}_{0}\right)
$$

Using the linearity of the Poisson equation, we can write $\delta \Phi(\mathbf{x})$ as

$$
\nabla[\varepsilon(\mathbf{x}) \nabla(\delta \Phi(\mathbf{x}))]=-4 \pi \delta \rho(\mathbf{x})
$$

where $\varepsilon(\mathbf{x})$ denotes the position-dependent permittivity. The difference in the electrostatic energy per unit cell between the two systems is

$$
\begin{aligned}
& \delta V\left(\mathbf{x}_{0}\right)=V_{1}\left(\mathbf{x}_{0}\right)-V_{2}\left(\mathbf{x}_{0}\right) \\
& =\frac{1}{2} \int_{V_{\text {box }}} d^{3} \mathbf{x}\left[\rho_{1}\left(\mathbf{x} ; \mathbf{x}_{0}\right) \Phi_{1}\left(\mathbf{x} ; \mathbf{x}_{0}\right)-\rho_{2}\left(\mathbf{x} ; \mathbf{x}_{0}\right) \Phi_{2}\left(\mathbf{x} ; \mathbf{x}_{0}\right)\right] \\
& =C+\frac{1}{2} \int_{V_{\text {box }}} d^{3} \mathbf{x}\left[\rho_{t}\left(\mathbf{x} ; \mathbf{x}_{0}\right) \delta \Phi(\mathbf{x})+\delta \rho(\mathbf{x}) \Phi_{t}\left(\mathbf{x} ; \mathbf{x}_{0}\right)\right] \\
& =C+\int_{V_{\text {box }}} d^{3} \mathbf{x} \rho_{t}\left(\mathbf{x} ; \mathbf{x}_{0}\right) \delta \Phi(\mathbf{x})
\end{aligned}
$$

For the second to last equality, eqs 12 and 13 were used, as well as the linearity of the Poisson equation, allowing one to introduce the potential $\Phi_{t}\left(\mathbf{x} ; \mathbf{x}_{0}\right)$ generated by the charge density $\rho_{t}\left(\mathbf{x} ; \mathbf{x}_{0}\right)$. The constant $C$ is independent of $\mathbf{x}_{0}$ and can therefore not be detected when comparing the PMFs of the two systems. The two terms in the integral of eq 17 are equal, as can be shown using eq 14 and two times integration by parts.

Equation 18 has important consequences: First, the effect of an implicit background charge, as introduced by the Ewald equations for a non-neutral system (eqs 3 and 9), is determined solely by the difference in charge densities between a nonneutral system with a neutralizing background charge, and a net neutral system with a realistic distribution of explicit counterions. Second, the effect on the electrostatic energy in the box $\left(\delta \mathrm{V}\left(\mathbf{x}_{0}\right)\right)$ can be computed from the electrostatic potential $(\delta \Phi(\mathbf{x}))$ generated by the difference between the two charge distributions $(\delta \rho(\mathbf{x})$; see eq 14$)$. For a point-charge test particle, which we considered here, eq 18 reduces to

$$
\delta V\left(\mathbf{x}_{0}\right)=C+q \delta \Phi\left(\mathbf{x}_{0}\right)
$$

Since we are only interested in the change of electrostatic energy as a function of the position $\mathbf{x}_{0}$ of the probe particle, the undetermined constant $C$ can be set to a convenient value (see below).

Analytic Expressions for Slab-like or Spherical Geometries. Here, we provide explicit expressions for eq 19 for (a) a low-dielectric slab surrounded by water, corresponding to a lipid membrane geometry, and (b) for a low-dielectric sphere in water, resembling a solvated globular protein or a solvated micelle.

Slab Geometry. Let $Q$ denote the nonzero total charge of a slab-like model system, including the charge of the test particle. The slab is oriented in the $x-y$ plane. In model systems 1 and $2, Q$ is either implicitly neutralized or explicitly neutralized by counterions, respectively. The explicit counterions are assumed to be homogeneously distributed in the water phase. The countercharge densities are thus given by

$$
\begin{aligned}
& \rho_{c, 1}(z)=\rho_{\mathrm{BG}}=-Q / V_{\mathrm{box}} \\
& \rho_{c, 2}(z)=-Q / V_{w}\left(1-\Theta_{l}(z)\right)
\end{aligned}
$$

where $V_{w}$ denotes the volume of the water phase, and $\Theta_{l}(z)$ is an indicator function that equals unity in the low-dielectric and zero in the water phase. The counter charge density difference is

$$
\delta \rho(z)=Q\left(1-\Theta_{l}(z)\right)\left[V_{w}^{-1}-V_{\text {box }}^{-1}\right]+\rho_{\mathrm{BG}} \Theta_{l}(z)
$$

Applying Gauss' law within the low-dielectric slab (of thickness $2 d$, centered around $z_{c}$ ) thus yields

$$
\begin{aligned}
& \delta V_{\text {slab }}\left(z_{0}\right)=2 \pi q \rho_{\mathrm{BG}}\left[d^{2}-\left(z_{0}-z_{c}\right)^{2}\right] / \varepsilon_{l}, \\
& \quad \text { if } \quad\left|z_{0}-z_{c}\right| \leq d
\end{aligned}
$$

corresponding to the text-book equation for the electrostatic potential across a charged slab. Here, $z_{0}$ is the position of the test charge $q$, and $\varepsilon_{l}$ denotes permittivity of the low-dielectric. The undetermined constant $C$ was chosen such that $\delta V_{\text {slab }}\left(z_{c} \pm\right.$ d) $=0$ at the water/hydrophobic interface. Because of the much higher permittivity of water, $\delta V \approx 0$ in the water phase. To express the energies in units common for MD simulations, an additional factor of $1 / 4 \pi \varepsilon_{0}$ must be applied to eq 23 , where $\varepsilon_{0}=$ $5.728 \times 10^{-4} e^{2} /(\mathrm{nm} \mathrm{kJ} / \mathrm{mol})$ is the vacuum permittivity.

We now apply the above analysis to quantify the effect of omitting explicit counterions in lipid membrane simulations. From eq 23 we computed $\delta V\left(\mathbf{x}_{0}\right)$ for a unit test charge $q=e$, while assuming a total charge of $Q_{\mathrm{BG}}=-1 e$ for the background charge. Figure $2 \mathrm{~A}$ presents $\delta V\left(z_{0}\right)$ for two common lipid membrane systems, as computed from eq 23. Here, we considered membranes of pure DMPC or pure POPE (128 lipid molecules plus 27.5 water molecules per lipid). The thickness $2 d$ of the hydrophobic core of the DMPC and the POPE membrane equals approximately 3.0 and $4.0 \mathrm{~nm}$, respectively. Compared to simulations with explicit counterions, the charge is stabilized by approximately 8 and $12 \mathrm{~kJ} / \mathrm{mol}$ at the center of the membrane, respectively. Thus, if (a) multiple non-neutralized ions are present, (b) the test particle carries multiple unit charges, or (c) a smaller lipid membrane patch is simulated, the stabilization may readily reach tens or 

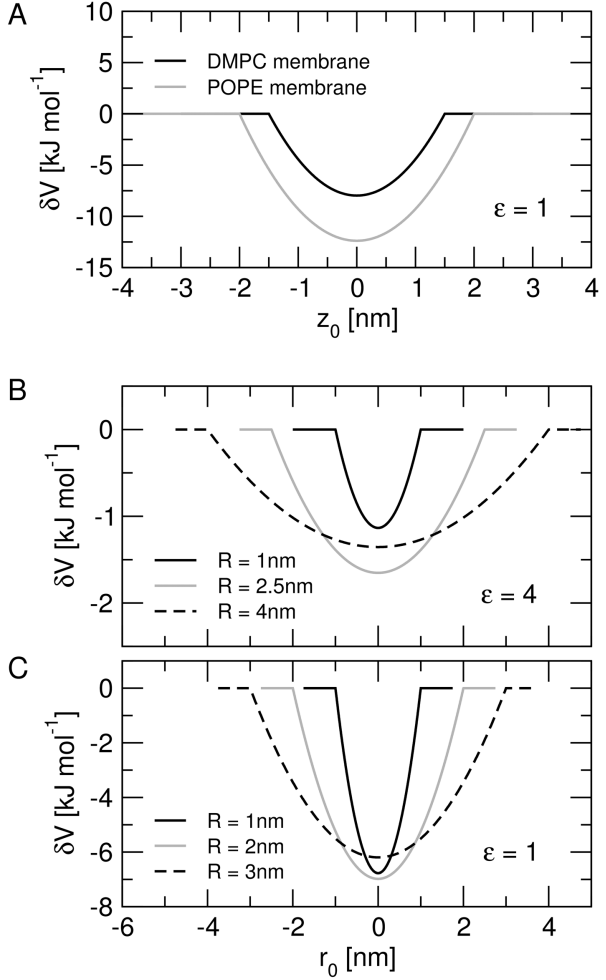

Figure 2. Effect on electrostatic potential of a unit test charge $q=e$ due a uniform background charge of $-e$; (A) for two different lipid membranes $(\varepsilon=1)$; (B) for three spherical protein of different radii $R$ model (see legend, $\varepsilon=4$ ); and (C) for a spherical micelle or oil droplet or different radii $R(\varepsilon=1)$.

hundreds of kilojoule per mole. Below, we compare the results of the analytical model to the results of the atomistic PMF calculations.

Spherical Geometry. Applying the analysis we used above for the slab geometry to a low-dielectric sphere of radius $R$ solvated in water yields

$$
\delta V_{\text {sphere }}\left(r_{0}\right)=2 \pi q \rho_{\mathrm{BG}}\left(R^{2}-r_{0}^{2}\right) / 3 \varepsilon_{l}, \quad \text { if } \quad r_{0} \leq R
$$

where $r_{0}$ is the distance of the test charge from the center of the sphere.

Figures $2 \mathrm{~B}$ and $\mathrm{C}$ present $\delta V\left(r_{0}\right)$ for spherical low-dielectrics of radius $R$ solvated in water, corresponding to a spherical protein (Figure $2 \mathrm{~B}, \varepsilon_{l}=4$ ) and to a spherical micelle or oil droplet (Figure 2C, $\varepsilon_{l}=1$ ). The curves were computed following eq 24, and the three box dimensions were assumed to equal $2 R+1.5 \mathrm{~nm}$, such that the protein would have a distance of $1.5 \mathrm{~nm}$ to the next periodic image. Here, the effect of the background charge is smaller because of the larger surface-tovolume ratio as compared to a slab geometry. For the protein, the effect is further reduced by the higher permittivity.

Before we end our discussion of the analytical model, we remark that the expressions derived here to quantify the artifact of the background charge for a slab and sphere geometry are related to the expressions proposed by others for correcting solvation free energies of ions in water. ${ }^{2,26,28-31}$ The difference, however, between our model and those correction terms is that while the correction terms were derived for a single ion in a periodic box with a homogeneous dielectric constant (water), we include the inhomogeneity of the system by using a position dependent dielectric constant.
PMFs for Neutral and Non-neutral Systems. Our analytical model predicts that the main consequence of using an implicit background charge to neutralize a heterogeneous system is an incorrect partitioning (or distribution) of mobile ions between high- and low-dielectric phases. To verify if this indeed occurs in MD simulations, we have computed the PMF for a test particle of charge $e / 4(e=|e|)$ across four hexadecane/ water slab systems with varying net charge. The PMF $G(z)$ is directly related to the partitioning via the Boltzmann factor: $\exp \left[-G(\mathrm{z}) / k_{\mathrm{B}} T\right]$, where $k_{\mathrm{B}}$ and $T$ denote the Boltzmann constant and the temperature, respectively. The total charge of the simulation box was $0,-0.75 e, 1.25 e$, and $2.25 e$ for systems i-iv (see Methods).

The PMFs for systems i-iv are shown in Figure 3A. Here, the flat region at $|z|>3 \mathrm{~nm}$ corresponds to bulk water, and $|z|<$
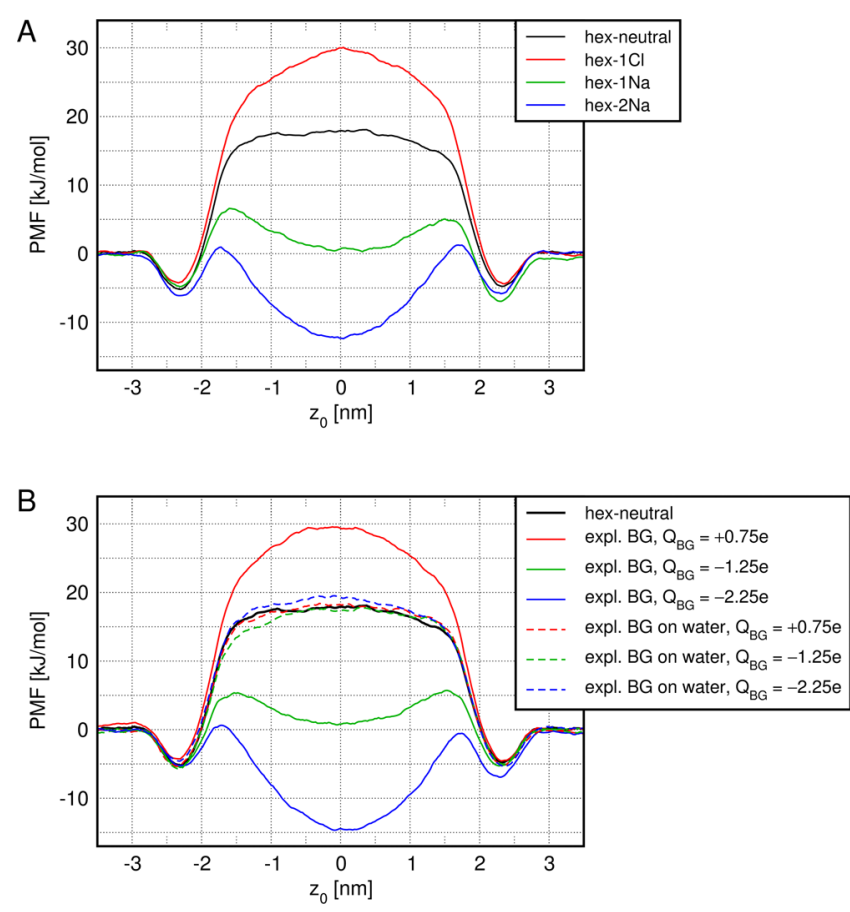

Figure 3. (A) PMFs of a test particle of charge $+e / 4$ across the hexadecane/water slab. Neutralized system (black) and non-neutral systems with one $\mathrm{Cl}^{-}$(red), one $\mathrm{Na}^{+}$(green), and two $\mathrm{Na}^{+}$ions (blue). (B) PMFs in explicitly neutralized systems. Solid lines: systems neutralized by altered partial charges of water and hexadecane atoms, corresponding to an explicit uniform background charge. Dashed lines: systems neutralized by altered partial charges of water atoms only, corresponding to a background charge only in the water phase.

$2 \mathrm{~nm}$ corresponds to positions inside the hexadecane. The PMFs demonstrate that non-neutralized charges in bulk water have indeed a drastic influence on the distribution of the test charge in the hexadecane/water system. In our test system, only two unit charges are sufficient to yield a PMF with the minimum at $z=0$, indicating a preferred position of the test particle at the center of the hexadecane slab (Figure 3A, blue curve). Comparing the PMFs shows that a charge difference of $1 e$ in bulk water induces a difference in the PMF by $\sim 15 \mathrm{~kJ} /$ mol. According to eq 23, the effect will be roughly four times stronger for a test particle that carries a full unit charge.

Effect of Spatial Distribution of Counter Charge. So far, the background charge was a purely mathematical term due to the omission of the $k=0$ term in the Ewald summation. To 

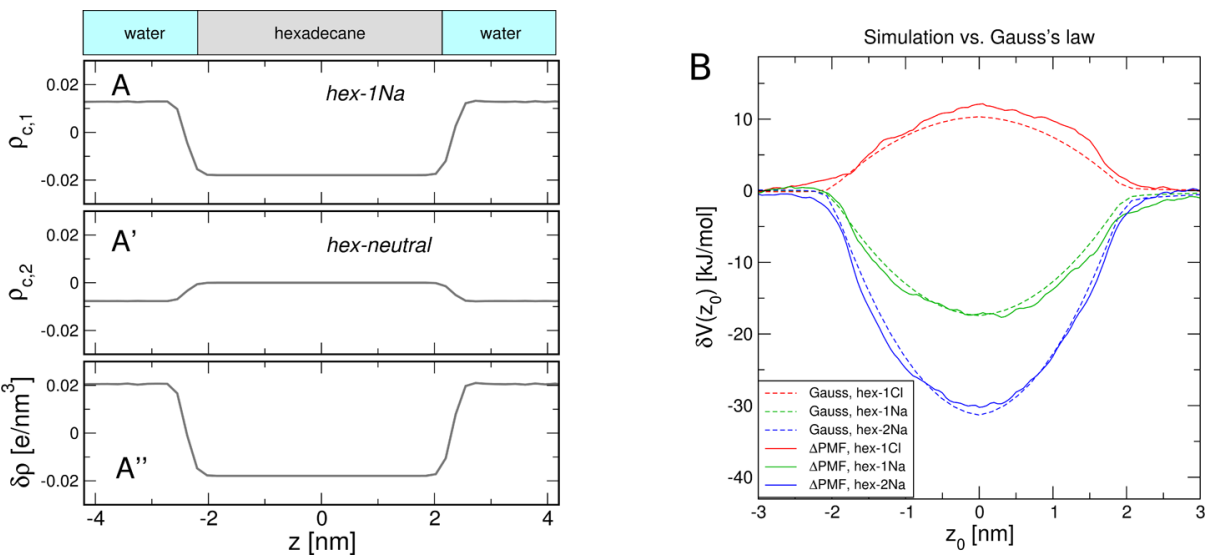

Figure 4. Analytic calculation of the background charge effect on PMFs using Poisson equation. (A) Counter charge density $\rho_{c, 1}(z)$ in the system $h e x-1 \mathrm{Na}$, composed of the explicit $\mathrm{Na}^{+}$ion in water $(|z|>2 \mathrm{~nm})$ plus the uniform background charge. $\left(\mathrm{A}^{\prime}\right) \rho_{c, 2}(z)$ in the neutral system hex-neutral, which is nonzero only in the water phase. ( $\left.\mathrm{A}^{\prime \prime}\right)$ Difference $\delta \rho(z)$ of counter charge density between the system hex-1Na and the system hex-neutral. (B) Potential energy difference $\delta V\left(z_{0}\right)$ between the three non-neutral systems (see legend) as compared to the neutral system. $\delta V\left(z_{0}\right)$ is plotted as a function of the test charge position $z_{0}$. Dashed lines: $\delta V\left(z_{0}\right)$ computed by applying the Poisson equation on $\delta \rho(z)$. Solid lines: Difference between the PMFs of the three charged systems as compared to the neutral system.

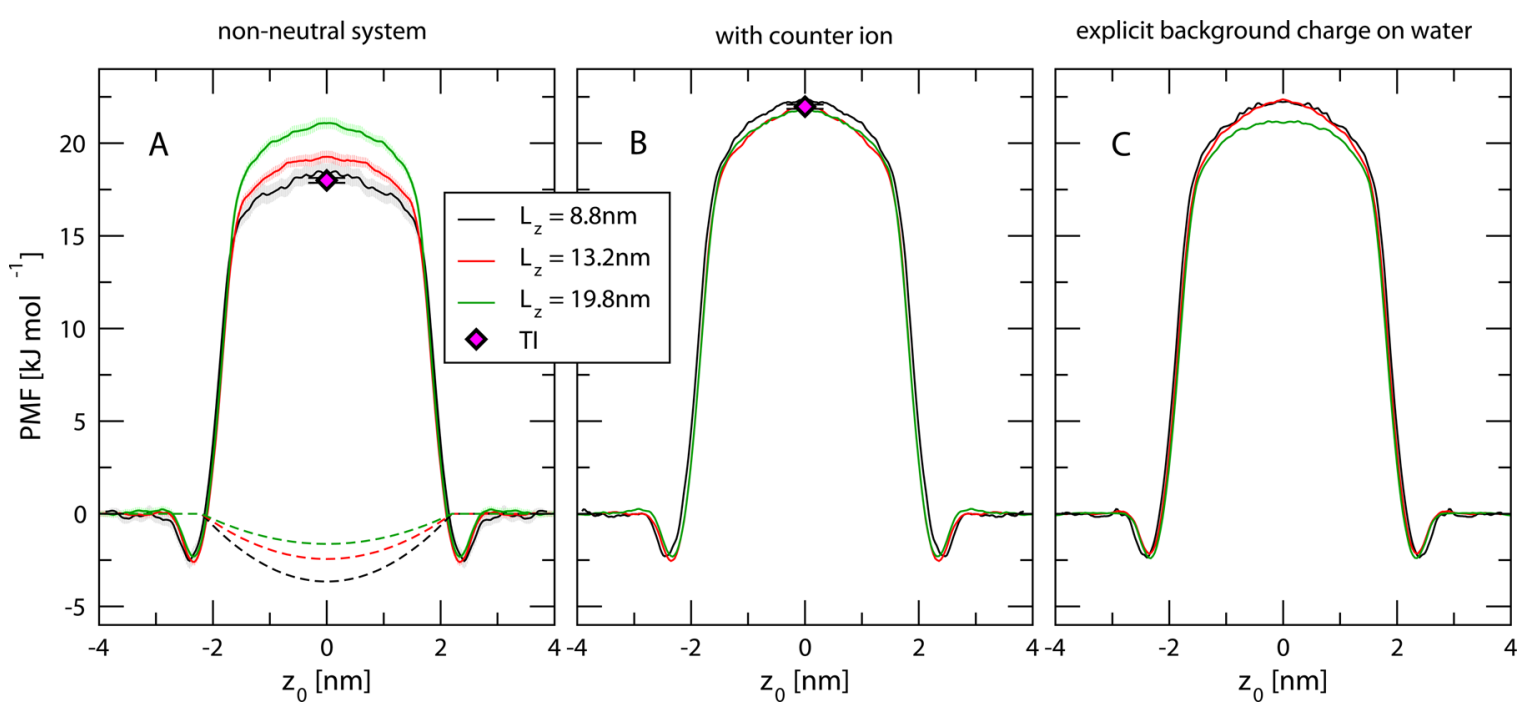

Figure 5. Self-interaction of an test charge (A) PMFs for the non-neutralized systems of different box heights $L_{z}$ of $8.8 \mathrm{~nm}$ (black), $13.2 \mathrm{~nm}$ (red), and $19.8 \mathrm{~nm}$ (green). PMFs are shown as solid lines with one SD as shaded area. The dashed lines show $\delta V\left(z_{0}\right)$ due to the stabilization of the test particle (charge $e / 4$ ) in the low-dielectric due to its own neutralizing background charge. (B) Respective PMFs with a counterion (charge $-e / 4$ ) in the bulk water, and (C) with an explicit background charge only in the water. Diamond: Free energy difference between hexadecane and water from thermodynamic integration.

investigate whether indeed the uniform background charge is responsible for the observed discrepancies in the PMFs, as well as to rationalize the effect of the implicit background charge in more intuitive (real-space) terms, we have computed two additional sets of PMFs in which we introduced an explicit background charge by adding small partial charges to the atoms in the system (Methods). We considered two situations. In the first, the counter charge is added to all atoms of the system, whereas in the second situation the counter charge is added to the water molecules only.

The PMFs for the systems with the explicit uniform background charge distributions are shown in Figure $3 \mathrm{~B}$ as solid lines. The PMFs are nearly identical to the PMFs of the non-neutral systems in Figure 3A, demonstrating that nonneutralized ions in the bulk water (Figure $3 \mathrm{~A}$ ) lead to similar results as an unphysical uniform distribution of the counter charge (Figure 3B, solid lines).
In contrast, the PMFs for systems in which an explicit background charge is present only inside the water phase (Figure 3B, dashed lines), are essentially identical to the PMF of the neutral system hex-neutral (Figure 3B, black). Taken together, the PMFs in figures 3A/B demonstrate that the spatial distribution of the counter charge (whether it is uniformly distributed over the whole box, or only over the water phase) has a large influence on the distribution of the charged test particle. In systems with an inhomogeneous dielectric constant, such as the hexadecane/water slab shown here, the uniform background charge therefore represents an unphysical counter charge distribution that causes severe artifacts on the partitioning of ions in the system.

Agreement with the Analytic Model. To illustrate the application of eqs 18 to 23 for our test charge, we show in Figure $4 \mathrm{~A}-\mathrm{A}^{\prime \prime}$ the charge density $\rho_{c, 1}(z)$ and $\rho_{c, 2}(z)$ for the systems hex-1Na and hex-neutral, respectively, as well as the 
difference between these densities, $\delta \rho(z)$. Via double integration of the Poisson eq 14 and using eq 18, we computed $\delta V\left(z_{0}\right)$ for a test charge $q=e / 4$ as a function of test particle position $z_{0}$. Here, we used a $z$-dependent permittivity $\varepsilon(z)=1$ $+79 \rho_{w}(z) / \rho_{w \text {,bulk, }}$ where $\rho_{w}(z)$ is the water density obtained from an equilibrium simulation of the hexadecane/water box and $\rho_{w, \text { bulk }}$ the density of bulk water (not shown). This choice for $\varepsilon(z)$ accounts for the permittivities of 1 and 80 inside the (nonpolarizable) hexadecane model and inside bulk water, respectively, and it yields a continuous average permittivity at the hexadecane-water interface due to surface fluctuations. In Figure $4 \mathrm{~B}$ we show the computed $\delta V\left(z_{0}\right)$ as dashed lines for the three charged systems. We note that directly applying the analytic expression for the slab (eq 23) yields a very similar result, yet the thickness of $2 d$ of membrane is not very accurately defined due to fluctuations of the water/hexadecane interface.

For comparison, we also plotted the differences between the each of the PMFs of the three non-neutral systems and the PMF of the neutral system as solid curves in Figure 4B. The latter were obtained by subtracting the black curve from the colored curves in Figure 3A. The excellent agreement demonstrates that the Poisson equation applied to $\delta \rho(z)$ correctly predicts the effect of omitting explicit counterions on the PMFs.

Effect of the Box Size and Self-Interaction of an Excess Charge. We have not yet considered the situation in which there is only the charged test particle, but no other charges around. According to the above analysis, we expect that omitting an explicit counterion would stabilize the charge in the low-dielectric, where the stabilization is proportional to the difference in charge density $\delta \rho(\mathbf{x})$ with respect to the explicitly neutralized system. Since the uniform density of the background charge is inversely proportional to the volume of the box, we have used the box volume as a parameter to control $\delta \rho(\mathbf{x})$. Thus, the three simulation systems contained the same number of hexadecane molecules but different number of water molecules. From a physics point of view, the PMFs for the three systems should be identical, because the test particle should not be influenced by water molecules far away from the hexadecane slab.

However, as shown in Figure 5A, the barrier height at the center of the hexadecane slab decreases with decreasing box size. The difference with respect to the explicitly neutralized system can again be rationalized by eqs 18 and 23: The smaller the box, the higher the density of the background charge inside the slab and, hence, the stronger the stabilization of the test charge in the low dielectric hexadecane slab. Application of eq 23 for the different box sizes yields $\delta V\left(z_{0}\right)$ of the test particle due to the omission of a counterion (Figure 5A, dashed lines). At the center of the membrane, the test particle is spuriously stabilized by $3.7,2.4$, and $1.6 \mathrm{~kJ} / \mathrm{mol}$ for the three box sizes, respectively, in good agreement with the differences between the PMFs. Theses findings demonstrate that the test particle is indeed artificially stabilized by its own background charge and that the Poisson equation applied to $\delta \rho(\mathbf{r})$ again predicts the strength of this effect. Note that since $\rho_{\mathrm{BG}}=-q / V_{\mathrm{box}}$ the effect is quadratic in the charge of the particle. Thus, for a test particle carrying one unit charge instead of $e / 4$, the effect would be 16 times stronger, causing a stabilizing potential between 26 and $59 \mathrm{~kJ} / \mathrm{mol}$.

For comparison, we have also computed the PMFs for the three box sizes with a counter charge $-e / 4$ on one of the water molecules. As shown in Figure 5B the respective PMFs are independent of the box volume, if the box is neutralized explicitly. Moreover, we have repeated the computation of the PMFs with an explicit background charge smeared out uniformly only over the water phase, as before. As shown in Figure 5C, these PMFs are also independent of the box size.

Agreement to Thermodynamic Integration (TI). The PMF calculations reported so far did not require to change the total charge of the system during the simulation. This is in contrast to simulations that aim to compute ionic solvation free energies or $\mathrm{p} K_{\mathrm{a}}$ values using, for instance, TI or free-energy perturbation. In such simulations, the system may either be kept neutral by generating a counterion, or by relying on the implicit background charge. Generating a counterion during TI obviously precludes the direct determination of the absolute solvation free energy of the sinlge test charge. However, it does allow to compute differences in the chemical potential between different parts of the simulation system. Employing the implicit background charge, in contrast, allows the calculation of absolute solvation free energies in homogeneous dielectrics because corrections for the artifacts are available. ${ }^{22,26,28-31}$

Here, we tested whether our analytical model holds equally for simulations that involve changing the net charge of the system. Using TI, we computed the solvation free energy of the test charge $(+e / 4)$ in the small simulation system. The test charge was either restrained at the center of the water phase (giving $\Delta G_{\mathrm{w}}$ ) or at the center of hexadecane slab (giving $\left.\Delta G_{\text {hex }}\right)$. The results are listed in Table 1 . When employing (a) a

Table 1. Solvation Free Energies in $\mathrm{kJ} / \mathrm{mol}$ for the Test Charge, and $\Delta \Delta G=\Delta G_{\text {hex }}-\Delta G_{\mathrm{w}}$

\begin{tabular}{lccc} 
& $\Delta G_{\mathrm{w}}$ & $\Delta G_{\text {hex }}$ & $\Delta \Delta G$ \\
counterion & $-54.9(2)$ & $-32.8(1)$ & $22.0(2)$ \\
background charge & $-3.97(1)$ & $14.0(1)$ & $18.0(2)$ \\
\hline
\end{tabular}

counterion or (b) the implicit background charge, TI yields $22.0(2)$ and $18.0(2) \mathrm{kJ} / \mathrm{mol}$, respectively (Figure $5 \mathrm{~A} / \mathrm{B}$, diamonds), in excellent agreement to the PMFs (Figure 5A/ $\mathrm{B}$, black curves). The agreement demonstrates that our analytic model is applicable to TI calculations that require a change of the total net charge.

\section{DISCUSSION}

We have derived an expression to quantitatively estimate the artifact due to the implicit background charge in Ewald simulations. With our model, we have addressed the question whether the background charge offers a realistic alternative for explicit counterions in MD simulations of charged systems. Although counterions offer a more realistic description, sampling problems associated with counterions have often been used to justify the use of the background charge instead. However, so far, it has been difficult to predict or correct the effect of the background charge on the results of the simulation, unless a single ion in a homogeneous box of solvent is considered. $^{22,26,28-31} \mathrm{We}$ found that the background charge affects the partitioning of charged particles in the simulation box if the system is not homogeneous. The cause of this artifact is the difference in the charge distribution between the real system, in which the counterions populate predominantly the higher dielectric, and the model system, in which the counter charge is uniformly distributed across the box. We derived an expression that quantifies the artifact in terms of this difference. 
With our analytical expression the effect of omitting explicit counterions can be computed analytically for simple geometries. The difference in charge density depends on both the total charge and box volume, as well as the relative volumes and geometries of the different dielectrics. For a typical lipid membrane simulation system containing 128 lipids plus water, our model predicts that a unit test charge would be artificially stabilized within the bilayer by $\sim 10 \mathrm{~kJ} / \mathrm{mol}$ for each nonneutralized unit charge in the system. In smaller systems, such as the hexadecane/water slab simulated here, or in systems containing many non-neutralized ions, the artificial stabilization can easily reach tens or even hundreds of kilojoules per mole.

Compared to lipid membrane simulations, the impact of the background charge for non-neutral protein simulations is smaller (i) because of the larger surface to volume ratio and (ii) because of the higher permittivity of proteins. For a spherical protein with $\varepsilon=4$, we estimate that a unit test charge is affected by 1 to $1.5 \mathrm{~kJ} / \mathrm{mol}$ at the center of the protein for each non-neutralized unit charge in the system. Our model thus provides a quantitative criterium for deciding if the background charge offers an acceptable trade-off between artifacts arising from sampling problems and artifacts arising from the homogeneous background charge distribution.

Several groups have documented artifacts in simulations using Ewald summation and suggested corrections. Some of these artifacts are due to the periodicity constraint and occur even in neutral systems, ${ }^{48,49}$ while other artifacts are found only if the system is charged. ${ }^{22,26,28-31}$ Ions in water are among the best studied cases and a number of corrections have been derived to correct the results of free energy calculations using Ewald summation. The first of these corrections removes the so-called self-energy, ${ }^{26}$ that is, the electrostatic interactions of the ion with its own periodic copies and the background charge:

$$
E^{\text {self }}=\frac{1}{2} \sum_{i} q_{i}^{2} \xi_{\mathrm{Ew}}
$$

with $\xi_{\mathrm{Ew}}$ a constant that depends on the box shape and dimension. $^{24}$ The second correction is a Born or PoissonBoltzmann term to represent the missing polarization in a finite and periodic simulation box..$^{25,27,29,31}$ The third correction corrects for the finite size of the ion. ${ }^{28,31}$

After correction, ionic hydration free energies obtained from simulations are in good agreement with experiment. However, these corrections have been derived only for the situation of a single (small) ion in water and therefore cannot be applied if the dielectric is not uniform throughout the simulation box. In addition, none of the correction terms contains an explicit dependency on the position of the charges. Therefore, including these corrections can only cause a uniform shift of the whole free-energy profile. Extending the corrections to inhomogeneous systems and including a dependency on the ion's position is not straightforward, but should contain terms that are similar to the ones derived here for a point charge in a low dielectric slab or sphere (eq 23 and 24, respectively).

Brooks and co-workers have proposed to correct Ewald artifacts in charged systems by subtracting the energy of a single particle carrying the total charge of the system and located in the same periodic box as the complete all-atom system. ${ }^{25}$ However, since this correction also does not depend on the ion's position, it cannot correct for the artifact in our free energy profiles, which we have shown to be strongly position dependent (eq 23).

Finally, Morgan and Massi have proposed a scheme to avoid contributions from the self-energy in free energy calculations that involve changing the charge of the system. ${ }^{50}$ In this scheme, transformations are constructed that conserve the norm of the total charge, as opposed to the total charge itself. Indeed, because the self-energy contributions depend quadratically on the total charge (eq 25), these contributions cancel. However, in this approach it is essential to sample a charged system, which can lead to an incorrect partitioning of charged particles, as we have shown here. Therefore, also in this approach the background charge will artifically compromise free energy calculations.

The model that we derived here can in principle also be used as a correction to improve the results of free energy computations that involve changing the charge of a single species inside the lower dielectric (e.g., protein or membrane), provided that any remaining ions remain inside the higher dielectric. As an example of the latter, we consider calculating the deprotonation free energy of an amino acid inside a protein by means of thermodynamic integration. The artificial stabilization of the deprotonated amino acid due to the background charge, which will overestimate the stability of the deprotonated state, can be estimated with eq 24 and subtracted from the final result. Alternatively, the sampling can be corrected if the analytical expressions for the excess energy due to the background charge are used to reweigh each configuration in an $\mathrm{MD}$ trajectory by a corrected Boltzmann factor. $^{33}$

Our approach of adding a background charge only to the water phase may also provide an alternative solution to neutralize the system. The presented examples show that such strategy can provide PMFs that are in much better agreement with simulations that have explicit counterions, as compared to simulations with an implicit uniform background charge. To make this approach more rigorous, one would first solve the Poisson-Boltzmann equation for the system and use the result to distribute the neutralizing background charge. Note, however, that by changing the partial charges on the water atoms, one changes the water force field. Therefore, the validity of this approach needs to be carefully verified by comparing the properties of the modified water model to those of the original model.

\section{CONCLUSION}

Simulating net charged systems is technically possible due to the omission of the $k=0$ term in the reciprocal contribution of the Ewald sum. However, whether the associated uniform background charge is an appropriate model for a converged distribution of counterions depends on the dielectric composition of the system. For heterogeneous systems with different dielectrics, such as proteins or membranes solvated in water, the uniform background charge can lead to strong artifacts in the chemical potential of charged particles. We traced the origin of this artifact to the difference in the charge distribution in the simulation box between the systems with a uniform background charge and with explicit counterions. Because a uniform charge density introduces no electrostatic gradient, it may seem paradoxical that the background charge can stabilize a non-neutralized charge inside the lower dielectric. However, because in reality counterions are always inside the higher dielectric, the counter charge distribution is 
nonuniform and therefore generates electrostatic gradients. The absence of such gradients when relying on the background charge to neutralize the system thus causes the artifact. We also derived an expression to quantify the impact of the background charge on the free energy profile of charged particles, which allows one to determine whether the use of background charge is appropriate, or whether explicit counterions are required to neutralize the simulation box.

\section{AUTHOR INFORMATION}

\section{Corresponding Authors}

*E-mail: jhub@gwdg.de.

*E-mail: gerrit.x.groenhof@jyu.fi.

\section{Notes}

The authors declare no competing financial interest.

\section{ACKNOWLEDGMENTS}

We thank Gerhard Hummer, Robert van Leeuwen, and Berk Hess for helpful discussions, and Floris Beulens for critically reading the manuscript. J.S.H. was supported by a Marie Curie Intra-European Fellowship within the 7th European Community Framework Programme and by the Deutsche Forschungsgemeinschaft (HU 1971/1-1). G.G. is supported by the Academy of Finland.

\section{REFERENCES}

(1) Warshel, A. Nature 1976, 260, 679-683.

(2) McCammon, J. A.; Gelin, B.; Karplus, M.; Wolynes, P. G. Nature 1976, 262, 325-326.

(3) Lindorff-Larsen, K.; Piana, S.; Dror, R.; Shaw, D. Science 2011, $334,517-520$.

(4) Lange, O.; Lakomek, N.; Farès, C.; Schröder, G.; Walter, K.; Becker, S.; Meiler, J.; Grubmüller, H.; Griesinger, C.; de Groot, B. Science 2008, 320, 1471-1475.

(5) Allen, M. P.; Tildesley, D. J. Computer Simulation of Liquids; Clarendon Press: Oxford, 1987, pp 156-162.

(6) Ewald, P. P. Ann. Phys. 1921, 64, 253-287.

(7) Deserno, M.; Holm, C. J. Chem. Phys. 1998, 109, 7678.

(8) Sagui, C.; Darden, T. Annu. Rev. Biophys. Biomol. Struct. 1999, 28, 155-179.

(9) De Leeuw, S.; Perram, J. Phys. A 1981, 107, 179-189.

(10) Perram, J.; Petersen, H.; DeLeeuw, S. Mol. Phys. 1988, 65, 875893.

(11) Hockney, R. W.; Eastwood, J. W. Computer Simulation Using Particles; McGraw-Hill: New York, 1981, pp 267-304.

(12) Darden, T.; York, D.; Pedersen, L. J. Chem. Phys. 1993, 98, 10089-10092.

(13) Essmann, U.; Perera, L.; Berkowitz, M. L.; Darden, T.; Lee, H.; Pedersen, L. G. J. Chem. Phys. 1995, 103, 8577-8592.

(14) York, D.; Yang, W. J. Chem. Phys. 1994, 101, 3298-3300.

(15) Toukmaji, A.; Sagui, C.; Board, J.; Darden, T. J. Chem. Phys. 2000, 113, 10913-10927.

(16) Sagui, C.; Pedersen, L.; Darden, T. J. Chem. Phys. 2004, 120, 73-87.

(17) Nam, K.; Gao, J.; York, D. J. Chem. Theory Comp. 2005, 1, 2-13.

(18) Chang, C.-M.; Shao, Y.; Kong, J. J. Chem. Phys. 2012, 136, 114112.

(19) Barnes, J.; Hut, P. Nature 1986, 324, 446-449.

(20) Greengard, L.; Rokhlin, V. J. Comput. Phys. 1987, 73, 325-332.

(21) Wu, X.; Brooks, B. J. Chem. Phys. 2005, 122, 044107.

(22) Figueirido, F.; DelBueno, G.; Levy, R. J. Chem. Phys. 1995, 103, 6133-6142.

(23) Ballenegger, V.; Arnold, A.; Cerdà, J. J. J. Chem. Phys. 2009, 131, 094107.

(24) Nijboer, B.; Ruijgrok, T. J. Stat. Phys. 1988, 53, 361-382.
(25) Bogusz, S.; Cheatham, T.; Brooks, B. J. Chem. Phys. 1998, 108, $7070-7084$

(26) Hummer, G.; Pratt, L. R.; García, A. E. J. Phys. Chem. 1996, 100, $1206-1215$.

(27) Figueirido, F.; DelBueno, G.; Levy, R. J. Phys. Chem. B 1997, $101,5622-5623$.

(28) Hummer, G.; Pratt, L.; Garcia, A. J. Chem. Phys. 1997, 107, 9275-9277.

(29) Hummer, G.; Pratt, L.; Garcia, A. J. Phys. Chem. A 1998, 102, $7885-7895$.

(30) Sakane, S.; Ashbaugh, H.; Wood, R. J. Phys. Chem. B 1998, 102, $5673-5682$

(31) Hünenberger, P.; McCammon, J. J. Chem. Phys. 1999, 110, 1856-1872.

(32) Hünenberger, P.; McCammon, J. Biophys. Chem. 1999, 78, 6988

(33) Kastenholz, M.; Hünenberger, P. J. Phys. Chem. B 2004, 108, 774-788.

(34) Herce, H.; Garcia, A. Proc. Natl. Acac. Sci. U.S.A. 2007, 26, 20805-20810.

(35) Torrie, G. M.; Valleau, J. P. Chem. Phys. Lett. 1974, 28, 578581

(36) Hub, J. S.; de Groot, B. L. Proc. Natl. Acad. Sci. U.S.A. 2008, 105, $1198-1203$.

(37) Jorgensen, W. L.; Chandrasekhar, J.; Madura, J. D.; Impey, R. W.; Klein, M. L. J. Chem. Phys. 1983, 79, 926-935.

(38) Berger, O.; Edholm, O.; Jähnig, F. Biophys. J. 1997, 72, 20022013.

(39) Hess, B.; Kutzner, C.; van der Spoel, D.; Lindahl, E. J. Chem. Theory Comput. 2008, 4, 435-447.

(40) Miyamoto, S.; Kollman, P. A. J. Comput. Chem. 1992, 13, 952962

(41) Hess, B. J. Chem. Theory Comput. 2008, 4, 116-122.

(42) Nosé, S. Mol. Phys. 1984, 52, 255-268.

(43) Hoover, W. G. Phys Rev A 1985, 31, 1695-1697.

(44) Parrinello, M.; Rahman, A. J. Appl. Phys. 1981, 52, 7182-7190.

(45) Kumar, S.; Bouzida, D.; Swendsen, R. H.; Kollman, P. A.; Rosenberg, J. M. J. Comput. Chem. 1992, 13, 1011-1021.

(46) Hub, J. S.; de Groot, B. L.; van der Spoel, D. J. Chem. Theory Comput. 2010, 6, 3713-3720.

(47) Hess, B. J. Chem. Phys. 2002, 116, 209-217.

(48) Luty, B.; Tironi, I.; Van Gunsteren, W. J. Chem. Phys. 1995, 103, 3014-3021.

(49) Kurtovic, Z.; Marchi, M.; Chandler, D. Mol. Phys. 1993, 78, $1155-1165$

(50) Morgan, B. R.; Massi, F. J. Chem. Theory Comput. 2010, 6, $1884-1893$ 